\title{
Computation of Molecular Spectra on a Quantum Processor with an Error-Resilient Algorithm
}

\author{
J. I. Colless, V. V. Ramasesh, D. Dahlen, M. S. Blok, and M.E. Kimchi-Schwartz \\ Quantum Nanoelectronics Laboratory, Department of Physics, \\ University of California, Berkeley, California 94720, USA; \\ and Center for Quantum Coherent Science, University of California, \\ Berkeley, California 94720, USA \\ J. R. McClean, ${ }^{\dagger}$ J. Carter, and W. A. de Jong \\ Computational Research Division, Lawrence Berkeley National Laboratory, \\ Berkeley, California 94720, USA \\ I. Siddiqi* \\ Quantum Nanoelectronics Laboratory, Department of Physics, \\ University of California, Berkeley, California 94720, USA, \\ Center for Quantum Coherent Science, University of California, Berkeley, California 94720, USA; \\ and Materials Science Division, Lawrence Berkeley National Laboratory, Berkeley, California 94720, USA
}

(Received 7 August 2017; published 12 February 2018)

\begin{abstract}
Harnessing the full power of nascent quantum processors requires the efficient management of a limited number of quantum bits with finite coherent lifetimes. Hybrid algorithms, such as the variational quantum eigensolver (VQE), leverage classical resources to reduce the required number of quantum gates. Experimental demonstrations of VQE have resulted in calculation of Hamiltonian ground states, and a new theoretical approach based on a quantum subspace expansion (QSE) has outlined a procedure for determining excited states that are central to dynamical processes. We use a superconducting-qubit-based processor to apply the QSE approach to the $\mathrm{H}_{2}$ molecule, extracting both ground and excited states without the need for auxiliary qubits or additional minimization. Further, we show that this extended protocol can mitigate the effects of incoherent errors, potentially enabling larger-scale quantum simulations without the need for complex error-correction techniques.
\end{abstract}

DOI: 10.1103/PhysRevX.8.011021

Subject Areas: Condensed Matter Physics,

Quantum Physics,

Quantum Information

\section{INTRODUCTION}

Quantum computing, the field of physics dedicated to harnessing quantum phenomena to process information, is rapidly progressing along the path from theoretical curiosity to practical technology. Recent experimental progress has been swift, with successful demonstrations of proof-ofconcept algorithms on a range of fledgling quantum

\footnotetext{
*To whom correspondence should be addressed. irfan_siddiqi@berkeley.edu

${ }^{\dagger}$ Present address: Google Inc., Venice, CA 90291, USA.

${ }^{\ddagger}$ Present address: MIT Lincoln Laboratory, Lexington, MA 02421, USA.

Published by the American Physical Society under the terms of the Creative Commons Attribution 4.0 International license. Further distribution of this work must maintain attribution to the author(s) and the published article's title, journal citation, and DOI.
}

processors composed of natural or engineered quantum spins [1-5]. However, even in leading architectures such as superconducting circuits and trapped ions, state-of-the-art systems comprise only few to tens of qubits - the quantum analog of classical bits-and are difficult to control with high precision. For gate-based quantum processors to be competitive with, or outperform their classical counterparts, both qubit number and gate fidelity must increase significantly [6,7]. Indeed, much of the field is currently focused on the design of a multiqubit architecture capable of demonstrating an unambiguous quantum speed-up over classical computers.

Recent theoretical advances suggest that a hybrid approach-judiciously dividing a computation between quantum and classical resources-will likely find utility in specific applications prior to the emergence of universal quantum computation [8-11]. One such example is calculating the ground-state energy of complex chemical systems, such as is often required in photovoltaics, biological reactions, and catalyst design. Based on the quantum 
variational principle- that the ground-state wave function of any Hamiltonian minimizes the expected energy [12] an iterative protocol, known as the variational quantum eigensolver (VQE) was developed [13]. This approach uses a classical optimization routine to minimize the expected energy of candidate wave functions, using the quantum hardware to evaluate the expected energy. Essentially, the $\mathrm{VQE}$ leverages the unique capacity of shallow quantum circuits to prepare entangled states from which efficient classical sampling is not known to be possible.

Essential ingredients of the VQE algorithm have recently been demonstrated on a variety of experimental platforms [13-19]. These initial experiments indicate a robustness to systematic control errors (so-called coherent errors) which would preclude fully quantum calculations, as well as a manageable scaling of quantum circuit depth with Hamiltonian complexity $[18,19]$. However, work to date has focused primarily on calculating molecular ground-state energies; while extending the VQE approach to find excited states has been demonstrated in the optical domain, it required additional qubits, complicated multiqubit control, and additional variational searches [18]. The characterization of excited states is crucial for the description of all phenomena involving significant electronic dynamics, which encompasses a broad class of systems of interest [20]. For example, charge transfer excited states in battery or photovoltaic materials or excitonic excited states in photosynthetic systems are required for the accurate description of dynamics and performance. Moreover, even in the case of more standard chemical reactions, the involvement of electronic excited states may be required for an accurate description of the process, with traditional examples such as photodissociation relying critically on this characterization [21,22].

Using a quantum processor comprising two superconducting transmon qubits in a 3D geometry with real-time classical optimization, we diagonalize the electronic structure Hamiltonian of the hydrogen molecule over a wide range of nuclear separations using a variational algorithm. We demonstrate, for the first time within such an architecture, the ability to calculate the full energy spectrum of a given Hamiltonian beyond just the ground state. In addition, we implement a recently developed theoretical extension to the VQE approach based on a quantum subspace expansion (QSE), establishing its ability to at least partially mitigate stochastic, incoherent errors [23], allowing us to attain near-chemical accuracy $\left(1.6 \times 10^{-3} \mathrm{H}\right)$ in the calculated energy spectrum. Our work shows how errors can be suppressed via additional measurements, potentially offering an alternative to more advanced error-correction techniques, which are typically difficult to implement and require large resource overhead. These methods can be directly transferred to other systems, such as planar superconducting platforms and ion traps, where significant numbers of qubits can be routinely controlled and read out [1,2,24-26], potentially paving the way for larger more complex quantum simulations in the near future.

\section{GENERAL APPROACH}

The electronic structure Hamiltonian, an operator on the space of electronic wave functions, is first cast into a form suitable for evaluation on a quantum processor. Specifically, the Hamiltonian is first projected onto a discrete set of molecular orbitals - we use the conventional STO-3G basis set [27], which constitutes a so-called minimal set in that it represents the minimum number of orbitals required to represent a given atomic shell. The resulting fermionic Hamiltonian $H_{F}$ is finally mapped onto a two-qubit Hamiltonian $H_{Q}$ [Supplemental Material (SM): Mapping of the $\mathrm{H}_{2}$ Hamiltonian to Qubits [28]]. The correspondence between qubit states and chemical basis states is shown in Fig. 1(a). For the hydrogen molecule, $H_{Q}$ takes the form

$H_{Q}=g_{0}+g_{1} \sigma_{z}^{1}+g_{2} \sigma_{z}^{2}+g_{3} \sigma_{z}^{1} \sigma_{z}^{2}+g_{4} \sigma_{y}^{1} \sigma_{y}^{2}+g_{5} \sigma_{x}^{1} \sigma_{x}^{2}$,

where $\sigma_{k}^{i}$ is the $k$ th Pauli operator on the $i$ th qubit, and the coefficients $\left\{g_{m}\right\}$, and thus the total Hamiltonian, are functions of $R$, the separation between the two hydrogen nuclei. For a given two-qubit state $|\psi\rangle$, prepared on the quantum processor, the expectation $\left\langle H_{Q}\right\rangle$ is evaluated through repeated measurements of Pauli correlators.

An outline of the VQE algorithm is depicted in Fig. 1(b) and consists of parametrizing a quantum circuit $U(\vec{\theta})$ to prepare an ansatz wave function $|\psi(\vec{\theta})\rangle$, evaluating the expectation $\left\langle\psi(\vec{\theta})\left|H_{Q}\right| \psi(\vec{\theta})\right\rangle$ termwise using a quantum processor, and then using a classical minimization algorithm to update parameters until a minimum, $\vec{\theta}_{\min }$, is found. The quantum state $\left|\psi\left(\vec{\theta}_{\text {min }}\right)\right\rangle$ then constitutes an approximation to the ground state of $H_{Q}$, with an estimated energy of $\left\langle\psi\left(\vec{\theta}_{\text {min }}\right)\left|H_{Q}\right| \psi\left(\vec{\theta}_{\text {min }}\right)\right\rangle$.

Once the VQE algorithm has converged on an estimate of the ground-state wave function, the quantum subspace expansion can be applied by measuring additional Pauli correlators that form an approximate matrix representation of $H_{Q}$ within an expanded subspace. This matrix can then be diagonalized classically to yield both low-lying excitedstate energies and a refined ground-state energy estimate [Fig. 1(c)]. If the expansion is chosen such that its dimension scales polynomially with system size, this classical matrix calculation is efficient [23]. The effectiveness of the QSE thus requires the existence of such a subspace which captures a significant amount of the excited-state support.

We expect that molecular excited energy levels differ from the ground state primarily by excitations which promote a single electron from an occupied to an unoccupied orbital. Therefore, to a good approximation, these states are linear combinations of $\left\{S_{1}: a_{i}^{\dagger} a_{j}\left|\psi_{\mathrm{GS}}\right\rangle\right\}$, where $a_{j}\left(a_{i}^{\dagger}\right)$ are fermionic annihilation (creation) operators for the electronic orbitals. While $S_{1}$ could serve as a subspace, a more natural 


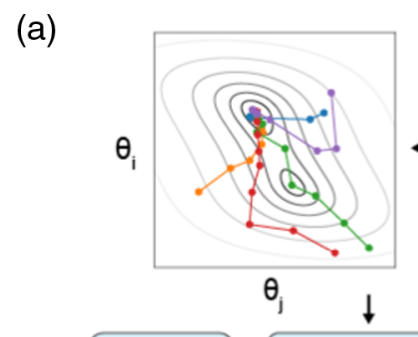

(b)

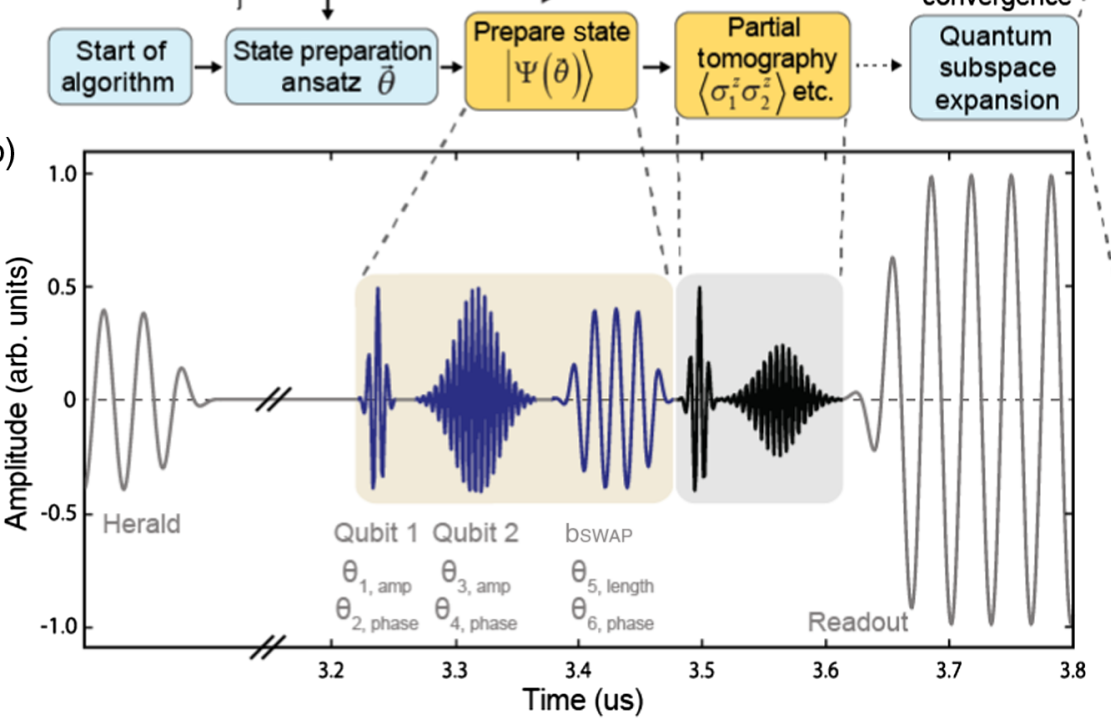

(c)

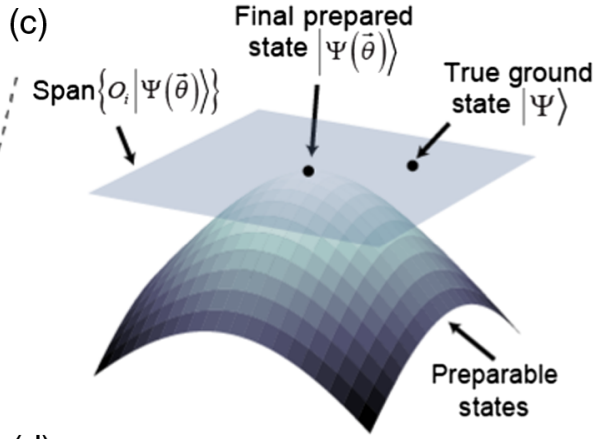

(d)

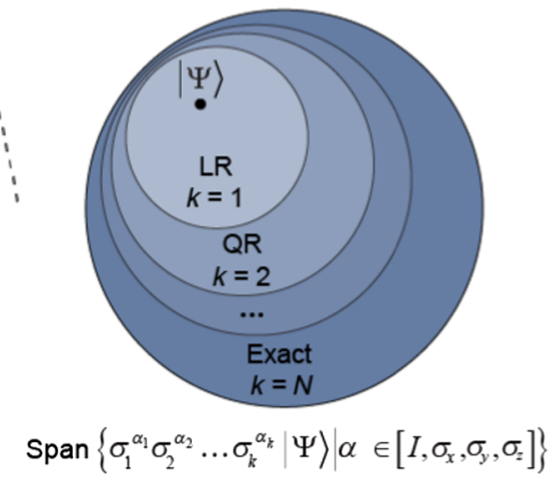

FIG. 1. Description of the variational quantum eigensolver algorithm and associated quantum subspace expansion. (a) Mapping between qubit states and electronic states of the hydrogen molecule in the STO-3G basis. As the molecular Hamiltonian preserves total spin projection, the four states with $s_{z}=0$ (dashed box) decouple from the other two. These four are mapped to the four two-qubit basis states. (b) Flow chart outline of the algorithm with classical resources colored in blue and quantum resources colored in yellow. Inset shows a cartoon example of the swarm minimization process with equal-energy contours shown in gray scale and 5 swarm particle trajectories (colored). (c) Cartoon of the QSE protocol; operators from $O_{i}$ are used to expand about the variational solution provided by the VQE, allowing for the mitigation of incoherent errors that otherwise render the true ground state inaccessible. (d) Typical qubit preparation and measurement sequence consisting of a projective heralding measurement (on which we later postselect so that the qubits start in the state $|00\rangle)$, single-qubit and two-qubit pulses, tomography pulses, and finally a projective readout.

choice when working with qubits involves the set of single Pauli flips $\left\{P_{1}: \sigma_{\alpha}^{k}\left|\psi_{\mathrm{GS}}\right\rangle \mid \alpha \in\{x, y, z\}, k \in\{1,2\}\right\}$, which we refer to as a linear response expansion. To calculate the matrix elements $H_{i j}$ in the $P_{1}$ basis, we use the quantum processor to evaluate the inner products,

$$
H_{i j}=\left\langle\psi_{\mathrm{GS}}\left|\sigma_{i}^{\dagger} H \sigma_{j}\right| \psi_{\mathrm{GS}}\right\rangle,
$$

where $\left|\psi_{\mathrm{GS}}\right\rangle$ is taken to be the initial approximate ground state $\left|\psi\left(\vec{\theta}_{\min }\right)\right\rangle$, found via the VQE routine.

While the nonlinear nature of the ansatz selected dictates that there may exist local minimum within the variational search portion of the procedure, in practice we utilize an initial guess that is thought to be within the basin of attraction for standard local minimizers of a high-quality minimum and multimodality is often not observed in practice [29]. In the case of excited states, we benefit from the linear construction of the problem, in that once the minimum and excitation operators have been selected, the solution of the linear subproblem is exact (within the subspace). Thus, for our procedure, local minima that can plague variational excitedstate searches are less likely to be an issue.
It was conjectured in Ref. [23] that in addition to providing a method for calculating molecular excitedstate energies the QSE could improve the accuracy of the initial VQE ground-state estimate. This is achieved through the addition of specific measurement operators which effectively increases the number of states under consideration. While the VQE can in principle correct for the presence of coherent gate errors, the QSE was thought to additionally correct for incoherent errors, such as dephasing or amplitude damping (SM: QSE with Errors [28]). As we discuss in Sec. IV, we find experimental support for this conjecture.

\section{EXPERIMENTAL METHODS}

\section{A. Quantum}

The quantum processor we use to evaluate expectation values consists of two superconducting qubits of the transmon variety $[30,31]$, one of the leading types of superconducting qubits in terms of design simplicity and coherence time. The qubits are initialized in the joint ground 
state $|00\rangle$ via a heralding measurement [32]. A generating circuit $U(\vec{\theta})$ is then used to prepare the desired trial wave function (with $\vec{\theta}$ specified by the classical hardware; see next section).

$U(\vec{\theta})$ consists of three microwave pulses resonant with the desired qubit transition [shown in Fig. 1(d)]. First, two single-qubit rotations take place, parametrized by amplitudes $\left(\theta_{1}, \theta_{3}\right)$ and phases $\left(\theta_{2}, \theta_{4}\right)$. Second, an entangling operation, known as the bSWAP gate [33], performs a rotation within the subspace spanned by $\{|00\rangle,|11\rangle\}$, parametrized by a length $\left(\theta_{5}\right)$ and a phase $\left(\theta_{6}\right)$.

Single-qubit pulses on qubit $A$ and $B$ last 50 and $70 \mathrm{ns,}$ respectively, and achieve fidelities of $\sim 99 \%$. The two-qubit pulse takes up to $310 \mathrm{~ns}$ and approaches a fidelity of $\sim 96 \%$. After state preparation via $U(\vec{\theta})$, tomographic reconstruction is used to evaluate $\langle H\rangle=\sum_{i j} h_{i j}\left\langle\sigma_{i} \sigma_{j}\right\rangle$. Note that in future implementations of VQE on larger quantum systems, full tomography will be impossible (due to an exponential scaling of the number of required measurements). Instead, only the necessary correlators will be directly measured. For this reason, our reconstruction of the two-qubit density matrix from the tomographic measurements did not use any method such as maximum-likelihood estimation which enforces physicality (positivity and trace normalization) on the result (see SM: Experimental Details [28]).

A near-quantum-limited traveling wave parametric amplifier [34,35] enables high-fidelity single-shot measurement of the joint qubit state (SM: Experimental Details [28]). The entire sequence, including both state preparation and measurement, comprises less than $\sim 1.5 \mu \mathrm{s}$, below the coherence times of the qubits: $16 \mu \mathrm{s} T_{1 A}, 13.5 \mu \mathrm{s} T_{2 A}^{*}$, $12 \mu \mathrm{s} T_{1 B}, 3.5 \mu \mathrm{s} T_{2 B}^{*}$.

\section{B. Classical}

With the two-qubit processor providing a means to efficiently evaluate $\langle H\rangle(\vec{\theta})$, the classical computer uses a particle-swarm optimizer (PSO) to find parameter values $\vec{\theta}_{\text {min }}$ which minimize this objective function, as shown in Fig. 2(a). Input parameters to the PSO are normalized to lie between 0 and 1. Amplitudes for each individual qubit are scaled by the magnitude required to pulse between the qubit's $|0\rangle$ and $|1\rangle$ state, and for the BSWAP by the magnitude required to pulse between the two-qubit $|00\rangle$ and $|11\rangle$ state. Pulse phases are normalized to lie between 0 and $2 \pi \mathrm{rad}$.

The PSO approach has two properties useful for this work: it is likely to avoid getting trapped in local minima and it is more robust to noisy objective-function calls [36]. The optimization treats a single point in parameter space as a particle, which has a velocity and position. A swarm of $n$ such particles $\left\{\left|\psi\left(\vec{\theta}_{s, i}\right)\right\rangle, i \in[1, n]\right\}$ (with $s$ the swarm iteration number) is first randomly initialized, and then at each iteration the particles' positions are updated based on both their own energy evaluation and those of others in
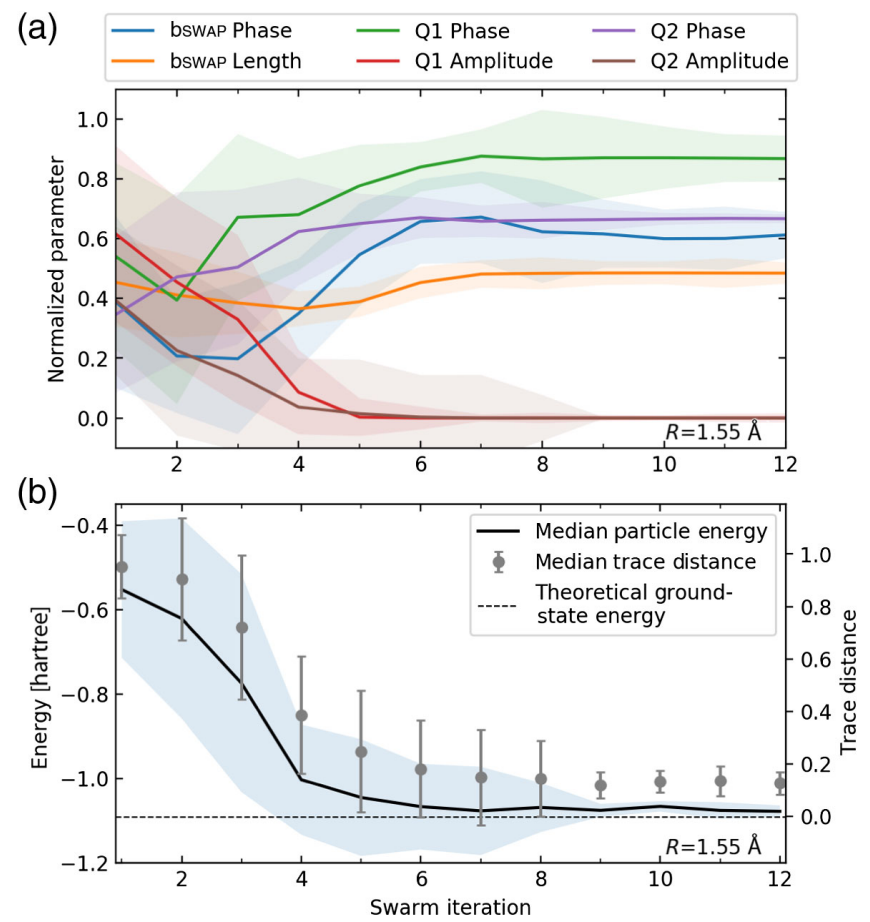

FIG. 2. Control parameter convergence as a function of classical optimizer iteration. (a) Median (solid line) and standard deviation (shaded region) for all six normalized parameter values as a function of swarm iteration number at an internuclear bond distance of $1.55 \AA$. A swarm of 20 particles demonstrates convergence after approximately 12 iterations or equivalently 240 function evaluations. See SM, VQE and Coherent Errors, for convergence details [28]. (b) Median energy (solid line) and standard deviation (shaded region) of swarm particles as a function of iteration number for the corresponding data in (a). Monotonic convergence of median energy towards the theoretical value is observed followed shortly thereafter by a rapid decrease in swarm energy variance. Trace distance (dots) of the variationally prepared ground state as a function of swarm iteration shows reasonable convergence to the theoretically expected ground state. The small remaining discrepancy is most likely due to the flat nature of the energy landscape and unavoidable decoherence during state preparation and readout.

the swarm (SM: Experimental Details [28]). Figure 2(b) shows how iterating through this loop allows the particles to converge on a set of control parameters that prepares the best approximation of our system's ground state $\left|\psi\left(\vec{\theta}_{g}\right)\right\rangle$ and its associated energy.

\section{RESULTS}

We apply our algorithm to the $\mathrm{H}_{2}$ molecule for 45 internuclear distances between 0.05 and $3.85 \AA$. As shown in Fig. 2(a) for an internuclear distance of $1.55 \AA$ and a random initialization of 20 swarm particles over $\vec{\theta}$, we observe good convergence of the control parameters within 12 swarm iterations. Each function evaluation consists of 10000 acquisitions and lasts on the order of a minute, resulting in a total algorithm run time of approximately $4 \mathrm{~h}$. 


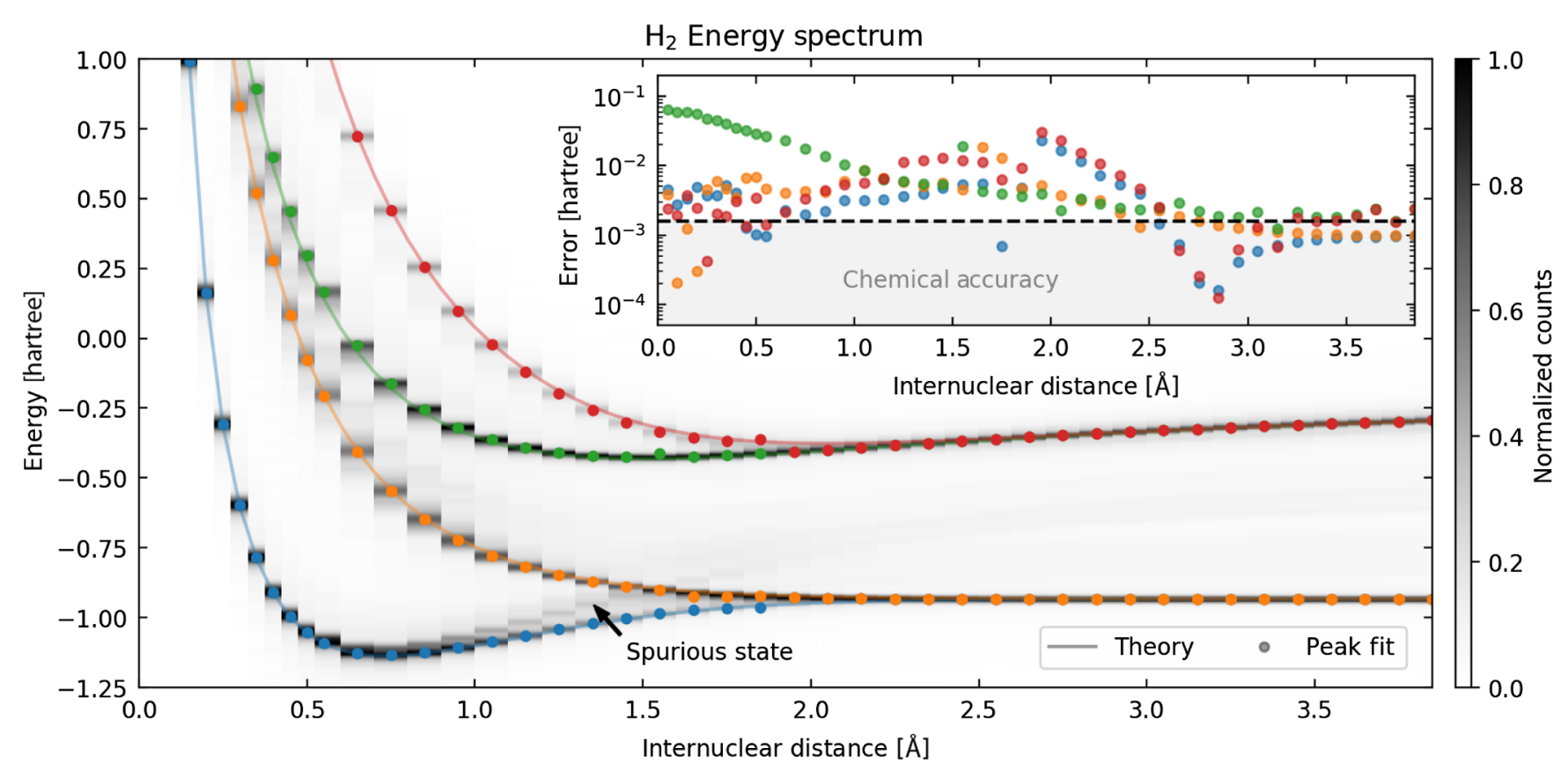

FIG. 3. $\mathrm{H}_{2}$ energy spectrum as a function of internuclear distance. Swarm particle energies for each bond length are histogrammed after application of a linear-response expansion and Gaussian filter. Energy estimates obtained by a peak finding routine are indicated by dots with theoretically predicted energy levels shown as solid lines. An unphysical spurious state emerges at internuclear distances greater than $\sim 1.2 \AA$ due to uncorrected incoherent errors. Inset shows errors in the estimated ground- and excited-state energies as compared to chemical accuracy $\left(1.6 \times 10^{-3} \mathrm{Ha}\right)$.

Experimentally optimized parameters show deviation from those that would be expected in the case of idealized gates (SM: VQE and Coherent Errors [28]). In particular, while the experimental single-qubit gate amplitudes and twoqubit bswAP length agree with numerical simulations, the phase of the bswAP differs significantly, most likely due to an unaccounted for Stark shift during application of the gate. The successful convergence of the algorithm despite this miscalibration thus provides additional proof of the protocol's intrinsic ability to correct for coherent errors.

Plotting the median energy of the swarm as a function of iteration number, we observe a large initial energy error due to the random nature of the particle initialization, followed by an almost monotonic decrease towards the exact theoretical value. When calculating an estimate for a new internuclear distance, we exploit the smoothness of the parameter landscape and reinitialize the swarm particles around the minimum found in the preceding run, allowing them to vary by only $5 \%$ from their previous optimum values. This technique allows us to reduce the resources required to achieve convergence from the initial 50 particles and 25 iterations used at $3.85 \AA$ to only 20 particles and 6 swarm iterations for subsequent runs. Once each internuclear separation of interest has been processed, we have an initial approximation for the ground-state energy function of the $\mathrm{H}_{2}$ molecule.

To derive excited states from this approximate ground state, we apply the linear-response QSE to each individually reconstructed density matrix recovered during the minimization process. The results of applying this expansion are plotted in Fig. 3 where data are binned with $1.5 \mathrm{mHa}$ resolution before convolution with a Gaussian filter (standard deviation of $7.5 \mathrm{mHa}$ ). Peak-finding routines are then used to estimate the mean energies for both the corrected ground and excited states. This shows improved robustness for small numbers of swarm iterations as it is less affected by outlying particles in the swarm yet to reach the global minimum. Extracting all four states reliably requires the use of the full linear-response expansion (SM: QSE and Choice of Expansion Operators [28]).

Errors between experimentally predicted energies for the ground and excited states and their true values are plotted in the inset of Fig. 3. Chemical accuracy, the level required to make realistic chemical predictions, is achieved for the ground and highest excited state across a wide range of internuclear distances. Estimates of the second and third excited-state energies are generally within an order of magnitude of this level. It is interesting to note that although the ground electronic state wave function near equilibrium requires little entanglement to accurately represent, the same is not true of the excited states. The QSE is able to approximate these states with only additional local measurements and efficient classical computation, without an increase in required entanglement on the quantum state of the qubits.

Figure 4 shows the deviations from the theoretically expected values for the corrected ground-state energies when using different underlying measurement operators for the applied QSE. Those involving just a single Pauli 


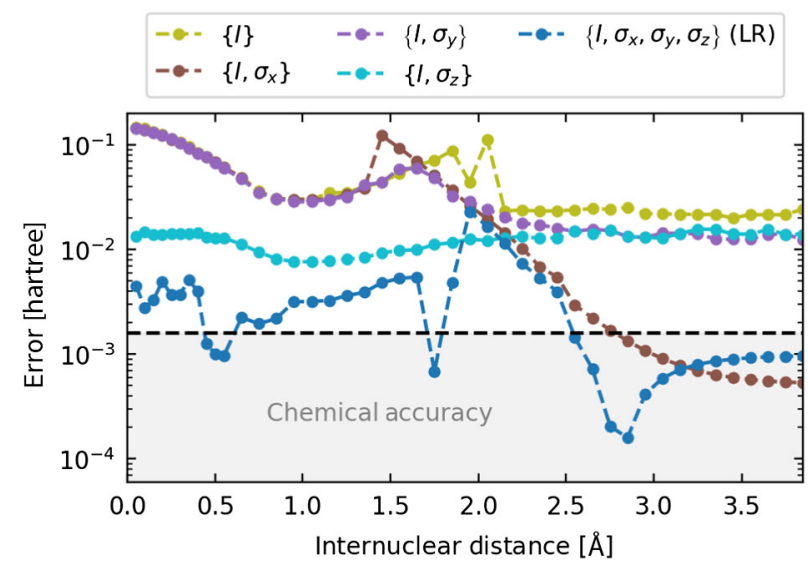

FIG. 4. Comparison of errors in the ground-state energy estimate when applying the QSE protocol using various combinations of expansion operators. The linear response expansion (dark blue dots) provides an improvement of more than an order of magnitude over the bare VQE estimate (yellow dots) for the majority of internuclear distances computed.

operator offer sporadic improvement over the uncorrected case, with the $\sigma_{z}$ operator achieving best results at smaller internuclear separations while the $\sigma_{x}$ operator is most useful at larger ones. The complete linear-response expansion is able to mitigate incoherent errors for which the bare VQE algorithm is unable to compensate and produces a reduction in the energy estimate error of almost 2 orders of magnitude over the entire range. By expanding the set of QSE operators (SM: QSE beyond Linear Response [28]), it is possible to further improve the ground-state estimate. However, such an expansion incurs increased computational cost. Going forward, it will be important to explore the trade-off between accuracy and QSE subspace size in extracting the spectra of larger molecules.

Note that ideally, the total number of extracted energy levels should be upper bounded by the dimension of the Hamiltonian. However, if the extant error channels cause the prepared VQE ground state to be sufficiently mixed (for a given set of QSE operators), it is possible to extract additional "spurious" energy levels. Such a spurious state is observed as indicated in Fig. 3 for internuclear distances between $\sim 1.2$ and $\sim 1.7 \AA$. In some cases, these states may be discarded on the basis of continuity of the energy as a function of internuclear distance. Alternatively, these states can be removed by increasing the span of the QSE operators (at the cost of an increased tomographic measurement overhead). The exact conditions for the presence of a spurious state are currently being investigated.

\section{CONCLUSION}

We experimentally implement an augmented variational quantum eigensolver that uses a polynomial number of additional tomographic measurements to extract molecular excited-state energies and mitigate incoherent errors on the ground-state estimate. With the hydrogen molecule as a test case, we additionally confirm the intrinsic ability of the algorithm to correct for coherent gate errors when pulse properties are optimized directly. Used with classical particle swarm minimization routines well suited to highdimensional noisy environments, these techniques yield energy estimates with near-chemical accuracy. Our results highlight the potential of QSE to significantly reduce the need for more advanced error-correction techniques, thereby facilitating practical applications of near-term quantum hardware.

\section{ACKNOWLEDGMENTS}

Financial support for ongoing quantum circuit development was provided by ARO/LPS under Grant No. W911NF-15-1-0496 and for the current experiment by the Director, Office of Science, Office of Advanced Scientific Computing Research, of the U.S. Department of Energy under Contract No. DE-AC02-05CH11231.

[1] T. Monz, D. Nigg, E. A. Martinez, M. F. Brandl, P. Schindler, R. Rines, S. X. Wang, I. L. Chuang, and R. Blatt, Realization of a Scalable Shor Algorithm, Science 351, 1068 (2016).

[2] S. Debnath, N. M. Linke, C. Figgatt, K. A. Landsman, K. Wright, and C. Monroe, Demonstration of a Small Programmable Quantum Computer with Atomic Qubits, Nature (London) 536, 63 (2016).

[3] X.D Cai, C. Weedbrook, Z.-E. Su, M.-C. Chen, M. Gu, M.-J. Zhu, L. Li, N.-L. Liu, C.-Y. Lu, and J.-W. Pan, Experimental Quantum Computing to Solve Systems of Linear Equations, Phys. Rev. Lett. 110, 230501 (2013).

[4] E. Lucero et al., Computing Prime Factors with a Josephson Phase Qubit Quantum Processor, Nat. Phys. 8, 719 (2012).

[5] M. Takita, A. D. Córcoles, E. Magesan, B. Abdo, M. Brink, A. Cross, J. M. Chow, and J. M. Gambetta, Demonstration of Weight-Four Parity Measurements in the Surface Code Architecture, Phys. Rev. Lett. 117, 210505 (2016).

[6] D. Wecker, B. Bauer, B. K. Clark, M. B. Hastings, and M. Troyer, Gate-Count Estimates for Performing Quantum Chemistry on Small Quantum Computers, Phys. Rev. A 90, 022305 (2014).

[7] A. G. Fowler, M. Mariantoni, J. M. Martinis, and A. N. Cleland, Surface Codes: Towards Practical Large-Scale Quantum Computation, Phys. Rev. A 86, 032324 (2012).

[8] J. R. McClean, J. Romero, R. Babbush, and A. AspuruGuzik, The Theory of Variational Hybrid QuantumClassical Algorithms, New J. Phys. 18, 023023 (2016).

[9] J. Li, X. Yang, X. Peng, and C. P. Sun, Hybrid QuantumClassical Approach to Quantum Optimal Control, Phys. Rev. Lett. 118, 150503 (2017).

[10] B. Bauer, D. Wecker, A. J. Millis, M. B. Hastings, and M. Troyer, Hybrid Quantum-Classical Approach to Correlated Materials, Phys. Rev. X 6, 031045 (2016).

[11] T. T. Tran et al., A Hybrid Quantum-Classical Approach to Solving Scheduling Problems, in Proceedings of the Ninth 
Annual Symposium on Combinatorial Search, 2016 (AAAI, New York, 2016).

[12] D. Griffiths, Introduction to Quantum Mechanics, 2nd ed. (Cambridge University Press, Cambridge, 2016).

[13] A. Peruzzo, J. McClean, P. Shadbolt, M.-H. Yung, X.-Q. Zhou, P. J. Love, A. Aspuru-Guzik, and J. L. O'Brien, A Variational Eigenvalue Solver on a Photonic Quantum Processor, Nat. Commun. 5, 4213 (2014).

[14] J. Du, N. Xu, X. Peng, P. Wang, S. Wu, and D. Lu, NMR Implementation of a Molecular Hydrogen Quantum Simulation with Adiabatic State Preparation, Phys. Rev. Lett. 104, 030502 (2010).

[15] Y. Shen, X. Zhang, S. Zhang, J.-N. Zhang, M.-H. Yung, and K. Kim, Quantum Implementation of the Unitary Coupled Cluster for Simulating Molecular Electronic Structure, Phys. Rev. A 95, 020501 (2017).

[16] Y. Wang et al., Quantum Simulation of Helium Hydride Cation in a Solid-State Spin Register, ACS Nano 9, 7769 (2015).

[17] P. O`Malley et al., Scalable Quantum Simulation of Molecular Energies, Phys. Rev. X 6, 031007 (2016).

[18] R. Santagati et al., in Proceedings of Frontiers in Optics, 2017 (Optical Society of American, Washington, DC, 2017), Paper No. FM4E.2.

[19] A. Kandala, A. Mezzacapo, K. Temme, M. Takita, M. Brink, J. M. Chow, and J. M. Gambetta, Hardware-Efficient Variational Quantum Eigensolver for Small Molecules and Quantum Magnets, Nature (London) 549, 242 (2017).

[20] L. Serrano-Andrés and M. Merchán, Quantum Chemistry of the Excited State: 2005 Overview, J. Mol. Struct. 729, 99 (2005).

[21] B. J. Berne, in Classical and Quantum Dynamics in Condensed Phase Simulations, Proceedings of the International School of Physics (World Scientific Publishing Company, Singapore, 1998).

[22] T. Helgaker, P. Jorgensen, and J. Olsen, Molecular Electronic-Structure Theory (Wiley, New York, 2013).

[23] J. R. McClean, M. E. Kimchi-Schwartz, J. Carter, and W. A. de Jong, Hybrid Quantum-Classical Hierarchy for Mitigation of Decoherence and Determination of Excited States, Phys. Rev. A 95, 042308 (2017).

[24] C. Song et al., 10-Qubit Entanglement and Parallel Logic Operations with a Superconducting Circuit, Phys. Rev. Lett. 119, 180511 (2017).
[25] https://www.research.ibm.com/ibm-q/.

[26] J. Kelly et al., State Preservation by Repetitive Error Detection in a Superconducting Quantum Circuit, Nature (London) 519, 66 (2015).

[27] W. J. Hehre, R. Ditchfield, and J. A. Pople, Self-Consistent Molecular Orbital Methods. XII. Further Extensions of Gaussian-Type Basis Sets for Use in Molecular Orbital Studies of Organic Molecules, J. Chem. Phys. 56, 2257 (1972).

[28] See Supplemental Material at http://link.aps.org/ supplemental/10.1103/PhysRevX.8.011021 for comparison of QSE effectiveness using single Pauli operators vs complete linear-response.

[29] I. Shavitt and R. Bartlett, Many-Body Methods in Chemistry and Physics: MBPT and Coupled-Cluster Theory (Cambridge University Press, Cambridge, England, 2009).

[30] J. Koch, T. M. Yu, J. Gambetta, A. A. Houck, D. I. Schuster, J. Majer, A. Blais, M. H. Devoret, S. M. Girvin, and R. J. Schoelkopf, Charge-Insensitive Qubit Design Derived from the Cooper Pair Box, Phys. Rev. A 76, 042319 (2007).

[31] H. Paik et al., Observation of High Coherence in Josephson Junction Qubits Measured in a Three-Dimensional Circuit QED Architecture, Phys. Rev. Lett. 107, 240501 (2011).

[32] J. Johnson, C. Macklin, D. H. Slichter, R. Vijay, E. B. Weingarten, J. Clarke, and I. Siddiqi, Heralded State Preparation in a Superconducting Qubit, Phys. Rev. Lett. 109, 050506 (2012).

[33] S. Poletto et al., Entanglement of Two Superconducting Qubits in a Waveguide Cavity via Monochromatic TwoPhoton Excitation, Phys. Rev. Lett. 109, 240505 (2012).

[34] C. Macklin, K. O'Brien, D. Hover, M. E. Schwartz, V. Bolkhovsky, X. Zhang, W. D. Oliver, and I. Siddiqi, A NearQuantum-Limited Josephson Traveling-Wave Parametric Amplifier, Science 350, 307 (2015).

[35] K. O'Brien, C. Macklin, I. Siddiqi, and X. Zhang, Resonant Phase Matching of Josephson Junction Traveling Wave Parametric Amplifiers, Phys. Rev. Lett. 113, 157001 (2014).

[36] K. E. Parsopoulos and M. N. Vrahattis, Recent Approaches to Global Optimization Problems through Particle Swarm Optimization, Nat. Comput. 1, 235 (2002). 